Article

\title{
On New Solutions of Time-Fractional Wave Equations Arising in Shallow Water Wave Propagation
}

\author{
Rajarama Mohan Jena ${ }^{1}$, Snehashish Chakraverty ${ }^{1}$ (D) and Dumitru Baleanu ${ }^{2,3, *(1)}$ \\ 1 Department of Mathematics, National Institute of Technology, Rourkela 769008, India \\ 2 Department of Mathematics, Faculty of Art and Sciences, Cankaya University, Balgat, 06530 Ankara, Turkey \\ 3 Institute of Space Sciences, 077125 Magurele-Bucharest, Romania \\ * Correspondence: dumitru@cankaya.edu.tr
}

Received: 15 May 2019; Accepted: 31 July 2019; Published: 8 August 2019

\begin{abstract}
The primary objective of this manuscript is to obtain the approximate analytical solution of Camassa-Holm $(\mathrm{CH})$, modified Camassa-Holm $(\mathrm{mCH})$, and Degasperis-Procesi (DP) equations with time-fractional derivatives labeled in the Caputo sense with the help of an iterative approach called fractional reduced differential transform method (FRDTM). The main benefits of using this technique are that linearization is not required for this method and therefore it reduces complex numerical computations significantly compared to the other existing methods such as the perturbation technique, differential transform method (DTM), and Adomian decomposition method (ADM). Small size computations over other techniques are the main advantages of the proposed method. Obtained results are compared with the solutions carried out by other technique which demonstrates that the proposed method is easy to implement and takes small size computation compared to other numerical techniques while dealing with complex physical problems of fractional order arising in science and engineering.
\end{abstract}

Keywords: shallow water wave; Caputo derivative; Camassa-Holm equation; differential transform method

\section{Introduction}

Nonlinear phenomena are of significant importance in natural sciences and engineering. Most of our real-life problems are modeled through the use of nonlinear phenomena. In the present years, fractional calculus has become widespread because of its applications in mathematical biology, electrochemistry, and physics [1-8]. For example, the earthquake model [9] and traffic model [10] with fractional derivatives have been demonstrated. However, sometimes, it is challenging to find the exact and numerical solutions of these models. During the last few decades, several analytical and numerical approaches have been established for the solution of such types of models such as homotopy perturbation method (HPM) [10,11], homotopy perturbation transform method [12,13], homotopy analysis method (HAM) [14,15], Adomian decomposition method (ADM) [16,17], sine-cosine method [18] and transform method [19]. Recently, multi-dimensional diffusion equation of fractional order has been solved by Kumar et al. [20] by modified HPM (m-HPM). In this approach, parameter $\mathrm{p}$ has been presented to extend the solution in series form, whereas the nonlinear terms can be extended by using He's polynomial [21]. It has been observed that the computation of He's polynomial is complicated, and the main disadvantage of this approach is its complexity in that regard and enormous calculations.

Among all the listed method, FRDTM plays a vital role because it takes small size computation, easy to implement as compared to other techniques. It was first introduced and developed by Keskin and Oturanc [22]. It is a beneficial and powerful semi-analytical approach. By implementation of the FRDTM, 
many physical nonlinear problems can be solved easily. In this article, the nonlinear time-fractional Camassa-Holm (CH) equation is taken as $[23,24]$

$$
\begin{gathered}
\frac{\partial^{\alpha} \psi}{\partial t^{\alpha}}+2 c \frac{\partial \psi}{\partial x}-\frac{\partial^{3} \psi}{\partial x^{2} \partial t}+3 \psi \frac{\partial \psi}{\partial x}=2 \frac{\partial \psi}{\partial x} \frac{\partial^{2} \psi}{\partial x^{2}}+\psi \frac{\partial^{3} \psi}{\partial x^{3}} \\
\text { for } t>0, x \in \mathbb{R}, 0<\alpha \leq 1
\end{gathered}
$$

with initial condition (IC)

$$
\psi(x, 0)=g(x)
$$

The modified Camassa-Holm $(\mathrm{mCH})$ and Degasperis-Procesi (DP) equations are derived from modified $b$-equation [25] which is

$$
\frac{\partial \psi}{\partial t}-\frac{\partial^{3} \psi}{\partial x^{2} \partial t}+(b+1) \psi^{2} \frac{\partial \psi}{\partial x}=b \frac{\partial \psi}{\partial x} \frac{\partial^{2} \psi}{\partial x^{2}}+\psi \frac{\partial^{3} \psi}{\partial x^{3}}
$$

where $b$ is a positive integer.

The time-fractional $\mathrm{mCH}$ and time-fractional DP are derived from fractional modified $b$-equation which may be written as

$$
\frac{\partial^{\alpha} \psi}{\partial t^{\alpha}}-\frac{\partial^{3} \psi}{\partial x^{2} \partial t}+(b+1) \psi^{2} \frac{\partial \psi}{\partial x}=b \frac{\partial \psi}{\partial x} \frac{\partial^{2} \psi}{\partial x^{2}}+\psi \frac{\partial^{3} \psi}{\partial x^{3}}
$$

By substituting $b=2, b=3$ into Equation (1c), we obtain the time-fractional $\mathrm{mCH}$ equation and time-fractional DP equation, respectively. So, the nonlinear time-fractional $\mathrm{mCH}$ equation is written as $[11,24,25]$

$$
\frac{\partial^{\alpha} \psi}{\partial t^{\alpha}}-\frac{\partial^{3} \psi}{\partial x^{2} \partial t}+3 \psi^{2} \frac{\partial \psi}{\partial x}=2 \frac{\partial \psi}{\partial x} \frac{\partial^{2} \psi}{\partial x^{2}}+\psi \frac{\partial^{3} \psi}{\partial x^{3}}, \text { for } t>0, x \in \mathfrak{R}, 0<\alpha \leq 1
$$

with IC

$$
\psi(x, 0)=p(x)
$$

and the nonlinear time-fractional DP equation is given as $[11,24,25]$

$$
\frac{\partial^{\alpha} \psi}{\partial t^{\alpha}}-\frac{\partial^{3} \psi}{\partial x^{2} \partial t}+4 \psi^{2} \frac{\partial \psi}{\partial x}=3 \frac{\partial \psi}{\partial x} \frac{\partial^{2} \psi}{\partial x^{2}}+\psi \frac{\partial^{3} \psi}{\partial x^{3}}, \text { for } t>0, x \in \mathfrak{R}, 0<\alpha \leq 1
$$

with IC

$$
\psi(x, 0)=f(x)
$$

These three models are the unidirectional shallow water waves propagation over a flat bottom. Equation (1) is a shallow water wave equation and was initially determined as an estimation to the incompressible Euler equation and observed to be integrable with a Lax pair [26]. Equation (3) is the shallow-water dynamics model and found to be completely integrable. All the equations possess not only the peakon solutions but also the multi-peakon solutions [26]. Recently, Degasperis and Gaeta [27] have been examined the behavior of the DP equation with the help of the bifurcation theory of dynamical system. To the best of authors' knowledge, for the first time, the fractional-order three relevant wave equations have been studied by the present authors analytically using FRDTM.

This article is prepared as follows: In Section 2, the essential features of fractional calculus related to the titled problem are included. Fundamental theories of FRDTM are described in Section 3. In Sections 4-6, implementations of FRDTM on $\mathrm{CH}, \mathrm{mCH}$, and DP equation are incorporated respectively. Numerical results and discussion are discussed in Section 7. Lastly, a conclusion is given in Section 8. 


\section{Preliminaries}

Definition 1. The operator $D_{x}^{\alpha}$ of $u(x)$ in Riemann-Liouville $(R-L)$ sense is

$$
D^{\alpha} u(x)=\left\{\begin{array}{l}
\frac{d^{m}}{d x^{m}} u(x), \quad \text { written as } \\
\frac{1}{\Gamma(m-\alpha)} \frac{d^{m}}{d x^{m}} \int_{0}^{x} \frac{u(t)}{(x-t)^{\alpha-m+1}} d t, \quad m-1<\alpha<m
\end{array}\right.
$$

where $m \in Z^{+}, \alpha \in R^{+}$.

Definition 2. The operator $J_{x}^{\alpha}$ of $u(x)$ in $R-L$ sense is described as

$$
J^{\alpha} u(x)=\frac{1}{\Gamma(\alpha)} \int_{0}^{x}(x-t)^{\alpha-1} u(t) d t, t>0, \alpha>0
$$

Following Podlubny [1] we may have

$$
\begin{aligned}
& J^{\alpha} t^{n}=\frac{\Gamma(n+1)}{\Gamma(n+\alpha+1)} t^{n+\alpha} \\
& D^{\alpha} t^{n}=\frac{\Gamma(n+1)}{\Gamma(n-\alpha+1)} t^{n-\alpha}
\end{aligned}
$$

Definition 3. The operator $D_{x}^{\alpha}$ of $u(x)$ in the Caputo sense is defined as

$$
D^{\alpha} u(x)=\left\{\begin{array}{l}
\frac{1}{\Gamma(m-\alpha)} \int_{0}^{x} \frac{u^{m}(t)}{(x-t)^{\alpha-m+1}} d t, \quad m-1<\alpha<m \\
\frac{d^{m}}{d t^{m}} u(x), \quad \alpha=m
\end{array}\right.
$$

\section{Definition 4.}

$$
\begin{gathered}
\text { (a) } D_{t}^{\alpha} J_{t}^{\alpha} f(t)=f(t), \\
\text { (b) } J_{t}^{\alpha} D_{t}^{\alpha} f(t)=f(t)-\sum_{k=0}^{m} f^{(k)}\left(0^{+}\right) \frac{t^{k}}{k !}, \text { for } t>0 \text { and } m-1<\alpha \leq m,
\end{gathered}
$$

\section{FRDTM}

Definition 5. Fractional reduced differential transform of an analytic and continuously differentiable function $u(x, t)$ is defined by

$$
U_{k}(x)=\frac{1}{\Gamma(\alpha k+1)}\left[D_{t}^{\alpha k} u(x, t)\right]_{t=t_{0}} \text { for } k=0,1,2, \ldots
$$

Taking the inverse transform of $U_{k}(x)$ is defined as

$$
u(x, t)=\sum_{k=0}^{\infty} U_{k}(x)\left(t-t_{0}\right)^{\alpha k}
$$

From Equations (11) and (12), we have

$$
u(x, t)=\sum_{k=0}^{\infty} \frac{1}{\Gamma(\alpha k+1)}\left[D_{t}^{\alpha k} u(x, t)\right]_{t=t_{0}}\left(t-t_{0}\right)^{\alpha k}
$$


In particular, at $t_{0}=0$, we get

$$
u(x, t)=\sum_{k=0}^{\infty} U_{k}(x) t^{\alpha k}=\sum_{k=0}^{\infty}\left(\frac{1}{\Gamma(\alpha k+1)}\right)\left\{D_{t}^{\alpha k} u(x, t)\right\}_{t=0} t^{\alpha k}
$$

As such Table 1 incorporates fractional reduced differential transform of few standard functions.

Table 1. The major operation of fractional reduced differential transform method (FRDTM) [22].

\begin{tabular}{cc}
\hline Functional Form & Transformed Form \\
\hline$g(x, t)$ & $G_{k}(x)=\frac{1}{k !}\left[\frac{\partial^{k}}{\partial t^{k}} g(x, t)\right]$ \\
$w(x, t)=g(x, t)+h(x, t)$ & $W_{k}(x)=G_{k}(x)+H_{k}(x)$ \\
$w(x, t)=\alpha g(x, t)$ & $W_{k}(x)=\alpha G_{k}(x)$ \\
$w(x, t)=g(x, t) h(x, t)$ & $W_{k}(x)=\sum_{r=0}^{k} G_{k-r}(x) H_{r}(x)$ \\
$w(x, t)=\frac{\partial^{r}}{\partial t^{r}} g(x, t)$ & $W_{k}(x)=\frac{(k+r) !}{k !} G_{k+r}(x)$ \\
$w(x, t)=\frac{\partial}{\partial x} g(x, t)$ & $W_{k}(x)=\frac{\partial}{\partial x} G_{k}(x)$ \\
$w(x, t)=D_{t}^{p \alpha}(g(x, t))$ & $W_{k}(x)=\frac{\Gamma(1+(k+p) \alpha)}{\Gamma(1+k \alpha)} G_{k+p}$ \\
\hline
\end{tabular}

In order to explain the concept of FRDTM, let us consider the following equation in the operator form as

$$
L \psi(x, t)+R \psi(x, t)+N \psi(x, t)=h(x, t)
$$

with IC

$$
\psi(x, 0)=g(x)
$$

where $L=\frac{\partial^{\alpha}}{\partial t^{\alpha}}, R, N$ are linear, nonlinear operators and $h(x, t)$ is an inhomogeneous source term.

Using Table 1 and Equation (11), Equation (15) reduces to

$$
\frac{\Gamma(1+\alpha k+\alpha)}{\Gamma(1+\alpha k)} \psi_{k+1}(x)=H_{k}(x)-R \psi_{k}(x)-N \psi_{k}(x), \text { for } k=0,1,2 \ldots
$$

where $\psi_{k}(x)$ and $H_{k}(x)$ are the transformed forms of $\psi(x, t)$ and $h(x, t)$, respectively.

Appling FRDTM on IC, we obtain

$$
\psi_{0}(x)=g(x)
$$

Using Equations (17) and (18), $\psi_{k}(x)$ for $k=1,2,3, \ldots$ can be determined.

Then by taking the inverse transformation of $\left\{\psi_{k}(x)\right\}_{k=0}^{n}$ gives $n$-term approximate solution as

$$
\psi_{n}(x, t)=\sum_{k=0}^{n} \psi_{k}(x) t^{\alpha k}
$$

So, the analytical result of Equation (15) is written as $\psi(x, t)=\lim _{n \rightarrow \infty} \psi_{n}(x, t)$.

\section{Implementation of FRDTM on the $\mathrm{CH}$ Equation}

The time-fractional CH Equation (1) in an operator form as

$$
D_{t}^{\alpha} \psi+2 c D_{x} \psi-D_{x x t} \psi+3 \psi D_{x} \psi=2 D_{x} \psi D_{x x} \psi+\psi D_{x x x} \psi, 0<\alpha \leq 1,
$$

with IC

$$
\psi(x, 0)=g(x)
$$


Applying FRDTM on Equations (20) and (21), the following recurrence relation is obtained as

$$
\left\{\begin{array}{l}
\frac{\Gamma(1+\alpha k+\alpha)}{\Gamma(1+\alpha k)} \psi_{k+1}(x)=2 \sum_{i=0}^{k}\left(\frac{\partial \psi_{i}(x)}{\partial x} \frac{\partial^{2} \psi_{k-i}(x)}{\partial x^{2}}\right)+\sum_{i=0}^{k}\left(\psi_{i}(x) \frac{\partial^{3} \psi_{k-i}(x)}{\partial x^{3}}\right)-2 c \frac{\partial \psi_{k}(x)}{\partial x}+\frac{\partial^{3} \psi_{k}(x)}{\partial x^{2} \partial t} \\
-3 \sum_{i=0}^{k}\left(\psi_{i}(x) \frac{\partial \psi_{k-i}(x)}{\partial x}\right) \\
\psi_{0}(x)=g(x)
\end{array}\right.
$$

Solving Equation (22) we obtain

$$
\begin{gathered}
\psi_{1}(x)=\frac{1}{\Gamma(1+\alpha)}\left(g(x) g^{(3)}(x)-2\left(c+\frac{3}{2} g(x)-g^{(2)}(x)\right) g^{(1)}(x)\right) . \\
\psi_{2}(x)=\frac{1}{\Gamma(1+2 \alpha)}\left[\begin{array}{l}
(g(x))^{2} g^{(6)}(x)+7 g^{(1)}(x) g(x) g^{(5)}(x)+g^{(4)}(x) \\
\left(-4 c g(x)-6 g(x)^{2}+13 g(x) g^{(2)}(x)+8\left(g^{(1)}(x)\right)^{2}\right)+8 g(x)\left(g^{(3)}(x)\right)^{2} \\
-12 g^{(1)}(x)\left(g^{(3)}(x)\right)^{2}\left(c+\frac{11}{4} g(x)-\frac{11}{6} g^{(2)}(x)\right)+4\left(g^{(2)}(x)\right)^{3}+ \\
(-21 g(x)-8 c)\left(g^{(2)}(x)\right)^{2}-30\left(g^{(1)}(x)\right)^{2} g^{(2)}(x)+4 g^{(2)}(x)\left(c+\frac{3}{2} g(x)\right)^{2} \\
+12\left(g^{(1)}(x)\right)^{2}\left(c+\frac{3}{2} g(x)\right)
\end{array}\right] .
\end{gathered}
$$

Continuing the procedure, likewise, the rest of the components can be evaluated. So the approximate analytical solution of Equation (20) is

$$
\psi(x, t)=\sum_{n=0}^{\infty} \psi_{n}(x) t^{n \alpha} .
$$

\section{Implementation of FRDTM on the $\mathrm{mCH}$ Equation}

Consider Equation (2) in an operator form as

$$
D_{t}^{\alpha} \psi-D_{x x t} \psi+3 \psi^{2} D_{x} \psi=2 D_{x} \psi D_{x x} \psi+\psi D_{x x x} \psi
$$

with IC

$$
\psi(x, 0)=p(x),
$$

Using FRDTM on Equations (26) and (27), the following recurrence relation is obtained as

$$
\left\{\begin{array}{l}
\frac{\Gamma(1+\alpha k+\alpha)}{\Gamma(1+\alpha k)} \psi_{k+1}(x)=2 \sum_{i=0}^{k}\left(\frac{\partial \psi_{i}(x)}{\partial x} \frac{\partial^{2} \psi_{k-i}(x)}{\partial x^{2}}\right)+\sum_{i=0}^{k}\left(\psi_{i} \frac{\partial^{3} \psi_{k-i}(x)}{\partial x^{3}}\right)+\frac{\partial^{3} \psi_{k}(x)}{\partial x^{2} \partial t} \\
-3 \sum_{i=0}^{k} \sum_{j=0}^{i}\left(\psi_{j} \psi_{i-j} \frac{\partial \psi_{k-i}(x)}{\partial x}\right) \\
\psi_{0}(x)=p(x)
\end{array}\right.
$$

Solving the recurrence relation Equation (28), we get

$$
\begin{gathered}
\psi_{1}(x)=\frac{1}{\Gamma(1+\alpha)}\left[p(x) p^{(3)}(x)-3(p(x))^{2} p^{(1)}(x)+2 p^{(2)}(x) p^{(1)}(x)\right] . \\
\psi_{2}(x)=\frac{1}{\Gamma(1+2 \alpha)}\left[\begin{array}{l}
(p(x))^{2} p^{(6)}(x)+7 p^{(1)}(x) p(x) p^{(5)}(x)+p^{(4)}(x) \\
\left(-6(p(x))^{3}+13 p(x) p^{(2)}(x)+8\left(p^{(1)}(x)\right)^{2}\right)+8 p(x)\left(p^{(3)}(x)\right)^{2} \\
-48 p^{(1)}(x)\left(p^{(3)}(x)\right)^{2}\left((p(x))^{2}-\frac{11}{24} p^{(2)}(x)\right)+4\left(p^{(2)}(x)\right)^{3}+ \\
(-30 p(x))\left(p^{(2)}(x)\right)^{2}+9(p(x))^{2} p^{(2)}(x)-96 p(x)\left(p^{(1)}(x)\right)^{2} p^{(2)}(x) \\
+36(p(x))^{3}\left(p^{(1)}(x)\right)^{2}-12\left(p^{(1)}(x)\right)^{4}
\end{array}\right]
\end{gathered}
$$


Similarly, the rest of the components $\psi_{3}, \psi_{4}, \ldots$ can be evaluated. So the approximate analytical solution of Equation (26) is

$$
\psi(x, t)=\sum_{n=0}^{\infty} \psi_{n}(x) t^{n \alpha},
$$

\section{Implementation of FRDTM on the DP Equation}

Considering Equation (3) in operator form, we get

$$
D_{t}^{\alpha} \psi-D_{x x t} \psi+4 \psi^{2} D_{x} \psi=3 D_{x} \psi D_{x x} \psi+\psi D_{x x x} \psi,
$$

with IC

$$
\psi(x, 0)=f(x)
$$

Applying FRDTM to Equation (32), we obtain

$$
\left\{\begin{array}{l}
\frac{\Gamma(1+\alpha k+\alpha)}{\Gamma(1+\alpha k)} \psi_{k+1}(x)=3 \sum_{i=0}^{k}\left(\frac{\partial \psi_{i}(x)}{\partial x} \frac{\partial^{2} \psi_{k-i}(x)}{\partial x^{2}}\right)+\sum_{i=0}^{k}\left(\psi_{i} \frac{\partial^{3} \psi_{k-i}(x)}{\partial x^{3}}\right)+\frac{\partial^{3} \psi_{k}(x)}{\partial x^{2} \partial t} \\
-4 \sum_{i=0}^{k} \sum_{j=0}^{i}\left(\psi_{j} \psi_{i-j} \frac{\partial \psi_{k-i}(x)}{\partial x}\right)
\end{array}\right.
$$

Applying FRDTM to Equation (33), we get

$$
\psi_{0}(x)=f(x) .
$$

Substituting Equation (35) in Equation (34), the following recursive values of $\left(\psi_{n}\right)_{n=1}^{\infty}$ are obtained

$$
\begin{gathered}
\psi_{1}(x)=\frac{1}{\Gamma(1+\alpha)}\left[f(x) f^{(3)}(x)-4(p(x))^{2} p^{(1)}(x)+3 p^{(2)}(x) p^{(1)}(x)\right] . \\
\psi_{2}(x)=\frac{1}{\Gamma(1+2 \alpha)}\left[\begin{array}{l}
(f(x))^{2} f^{(6)}(x)+9 f^{(1)}(x) f(x) f^{(5)}(x)+f^{(4)}(x) \\
\left(-8(f(x))^{3}+18 f(x) f^{(2)}(x)+15\left(f^{(1)}(x)\right)^{2}\right)+11 f(x)\left(f^{(3)}(x)\right)^{2} \\
-72 f^{(1)}(x)\left(f^{(3)}(x)\right)\left((f(x))^{2}-\frac{5}{8} f^{(2)}(x)\right)+9\left(f^{(2)}(x)\right)^{3} \\
-48(f(x))^{2}\left(f^{(2)}(x)\right)^{2}+16(f(x))^{4} f^{(2)}(x)-168 f(x)\left(f^{(1)}(x)\right)^{2} f^{(2)}(x) \\
+64(f(x))^{3}\left(f^{(1)}(x)\right)^{2}-24\left(f^{(1)}(x)\right)^{4}
\end{array}\right] .
\end{gathered}
$$

Continuing in this manner $\psi_{3}, \psi_{4}, \ldots$ can be evaluated. So the approximate analytical solution of Equation (32) is

$$
\psi(x, t)=\sum_{n=0}^{\infty} \psi_{n}(x) t^{n \alpha}
$$

\section{Results and Discussion}

In this section, approximate solutions of displacement $\psi(x, t)$ for different values of $\alpha$ are calculated for different values of $t$ and $x$ at fixed $c=0.005$ and $k=0.5$. In Section 4, IC [24,28] is considered as $\psi(x, 0)=g(x)=(k+c) e^{-|x|}-c$ for showing the nature of the displacements of CH Equation (1). The solutions $\psi(x, t)$ for different values of $t, x$ and $\alpha$ are depicted in Figure 1a-d for the CH equation. In Section 5, we considered the IC [11] as $\psi(x, 0)=p(x)=-2 \operatorname{sech}^{2}\left(\frac{x}{2}\right)$, a particular case for viewing the behavior of the displacements. The numerical results of $\psi(x, t)$ for various values of $t, x$ and $\alpha$ are portrayed in Figure 2a-d for the mCH Equation (2). Similarly, in Section 6, the IC [11] is assumed as $\psi(x, 0)=f(x)=-\frac{15}{8} \operatorname{sech}^{2}\left(\frac{x}{2}\right)$ for presenting the nature of the displacements. The numerical solutions $\psi(x, t)$ for various values of $t, x$ and $\alpha$ are illustrated in Figure 3a-d for the DP Equation (3). Also, 
comparison tests have been included among the existing solution and the results of Zhang et al. [11] and Zhang et al. [28] in Figures 4-9. One may see from Tables 2-4 that approximate solutions solved by FRDTM are quite close to the solutions solved by Zhang et al. [11,28].

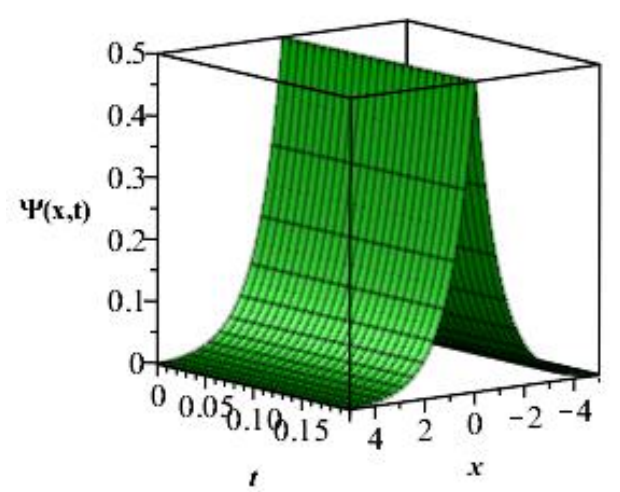

(a)

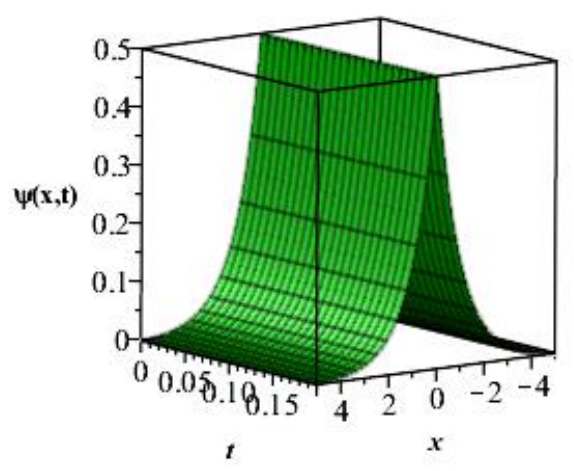

(c)

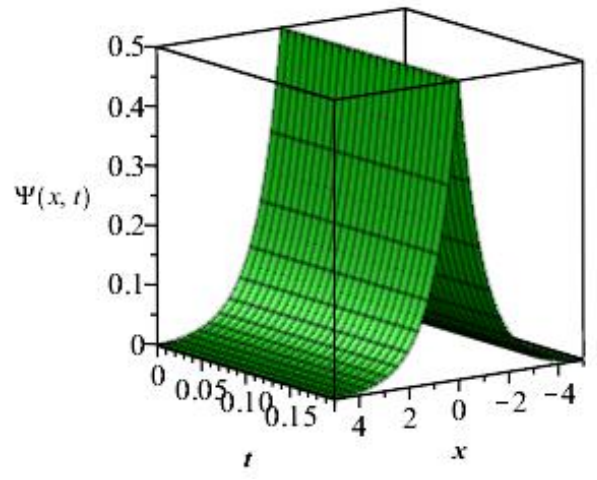

(b)

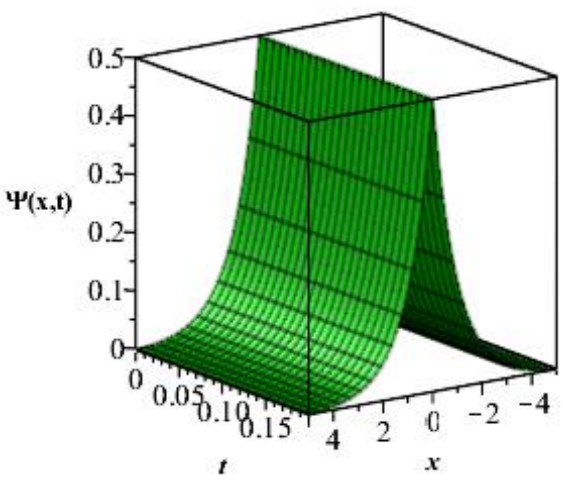

(d)

Figure 1. The solution plots of $\psi(x, t)$ at (a) $\alpha=0.5$ (b) $\alpha=0.25$ (c) $\alpha=0.75$ (d) $\alpha=1$ with $c=0.005$ and $k=0.5$ for Equation (25).

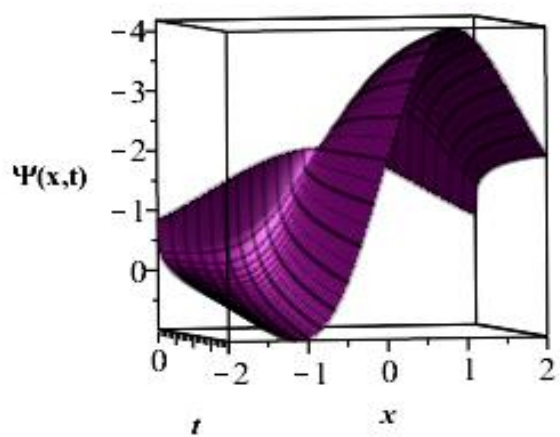

(a)

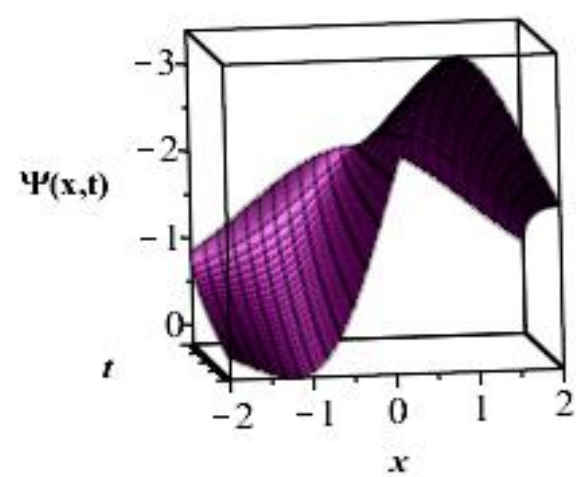

(b)

Figure 2. Cont. 


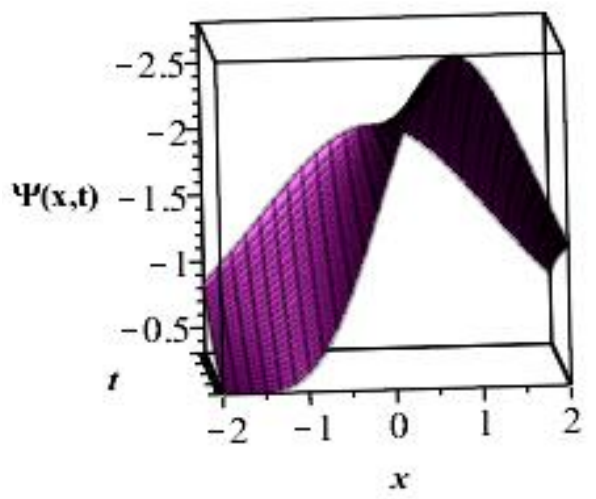

(c)

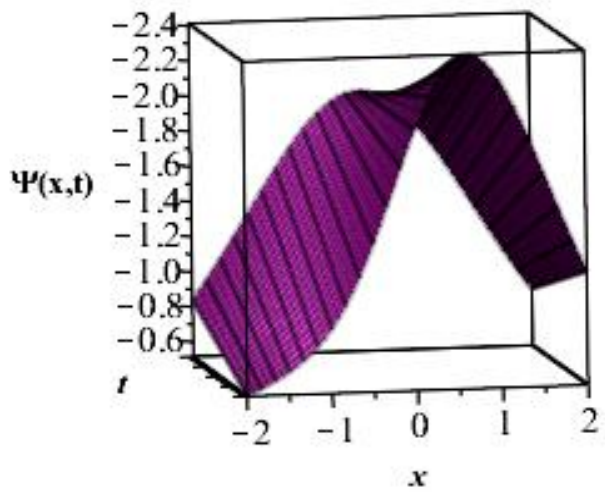

(d)

Figure 2. Plots of $\psi(x, t)$ with respect to $x$ and $t$ at (a) $\alpha=0.5$ (b) $\alpha=0.25$ (c) $\alpha=0.75$ (d) $\alpha=1$ for Equation (31).

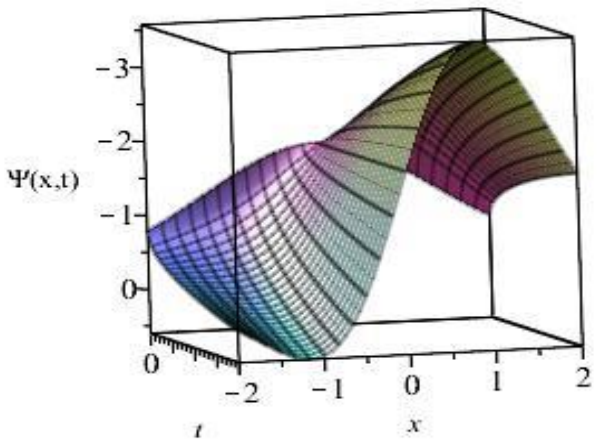

(a)

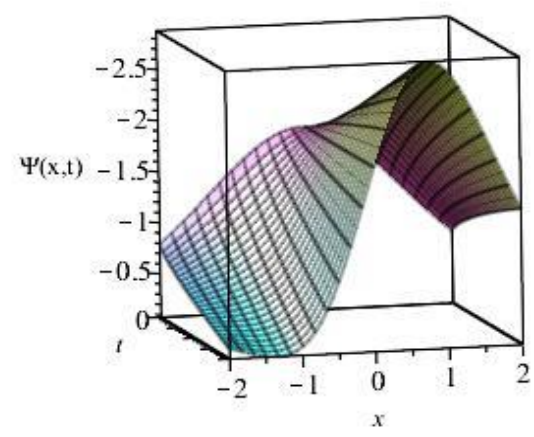

(c)

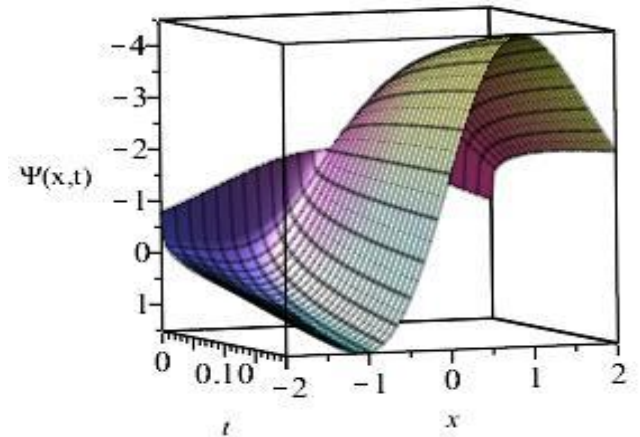

(b)

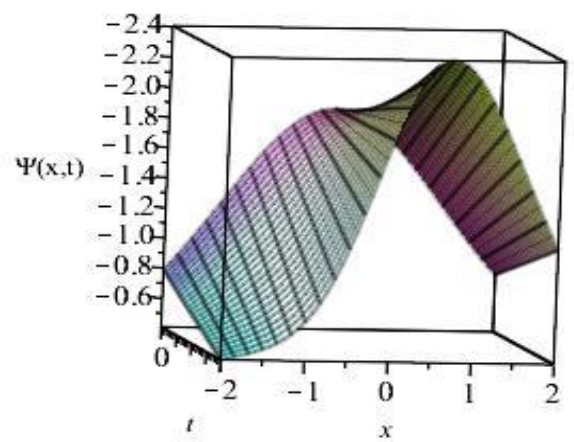

(d)

Figure 3. Plots of $\psi(x, t)$ with respect to $x$ and $t$ at (a) $\alpha=0.5$ (b) $\alpha=0.25$ (c) $\alpha=0.75$ (d) $\alpha=1$ for Equation (38). 


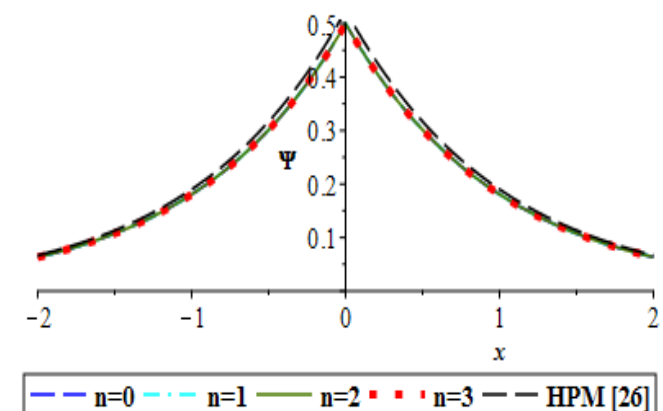

(a)

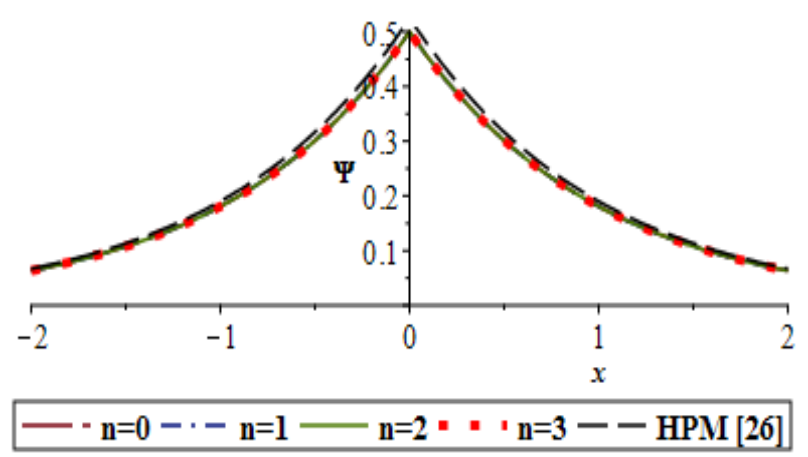

(b)

Figure 4. Comparison plots of $\psi_{F R D T M}$ and $\psi_{H P M}$ of Camassa-Holm $(\mathrm{CH})$ equation for $-2 \leq x \leq 2$. (a) $c=0.005, k=0.5, t=0.05, \alpha=1 ;(\mathbf{b}) c=0.005, k=0.5, t=0.1, \alpha=1$.

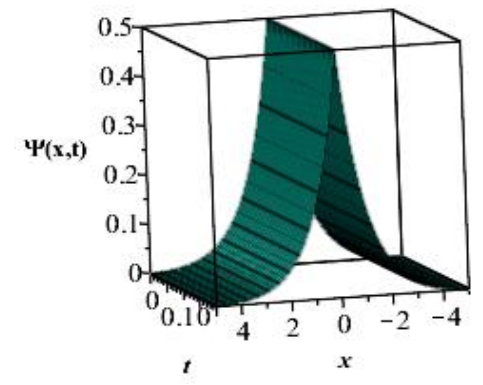

(a)

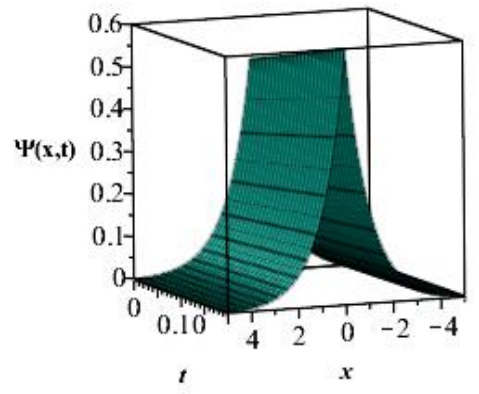

(b)

Figure 5. The solution plots of $\mathrm{CH}$ equation at $\alpha=1$ (a) two-terms FRDTM solution (b) homotopy perturbation method (HPM) solution [28].

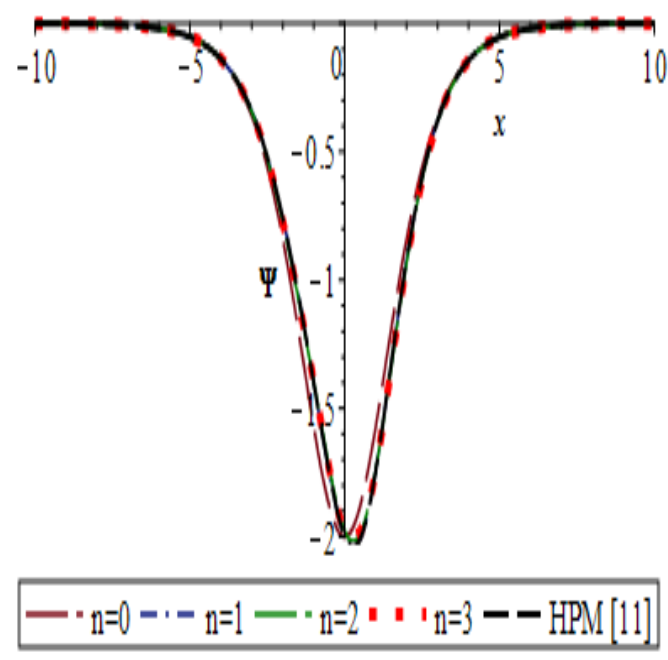

(a)

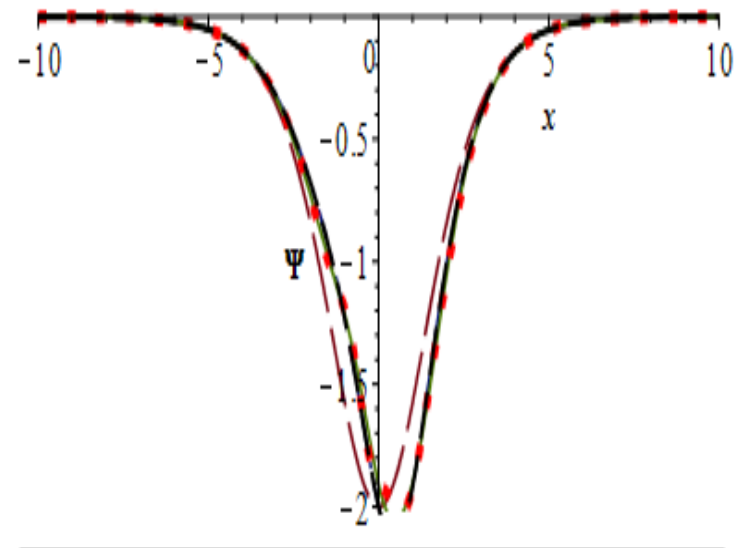

$\cdot n=0-\cdot+n=1-n=2 \cdot \cdot+1 n=3--H P M[11]$

(b)

Figure 6. Comparison plots of $\psi_{F R D T M}$ and $\psi_{H P M}$ of modified Camassa-Holm (mCH) equation for $-10 \leq x \leq 10$. (a) $t=0.05, \alpha=1$; (b) $t=0.1, \alpha=1$. 


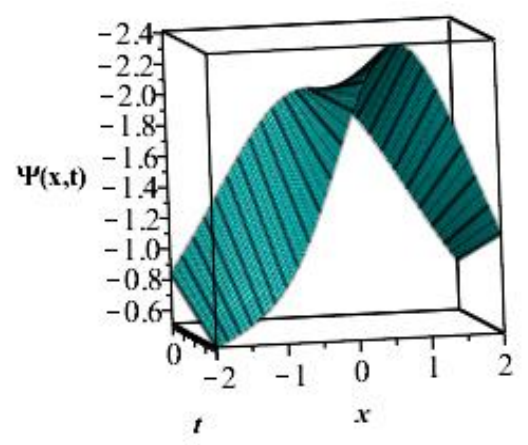

(a)

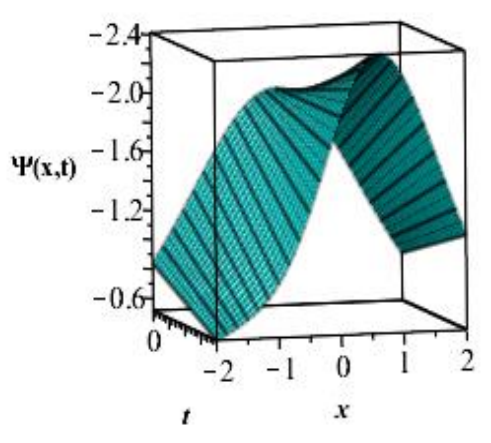

(b)

Figure 7. The solution plots of $\mathrm{mCH}$ equation at $\alpha=1$ (a) two-terms FRDTM solution (b) HPM solution [11].

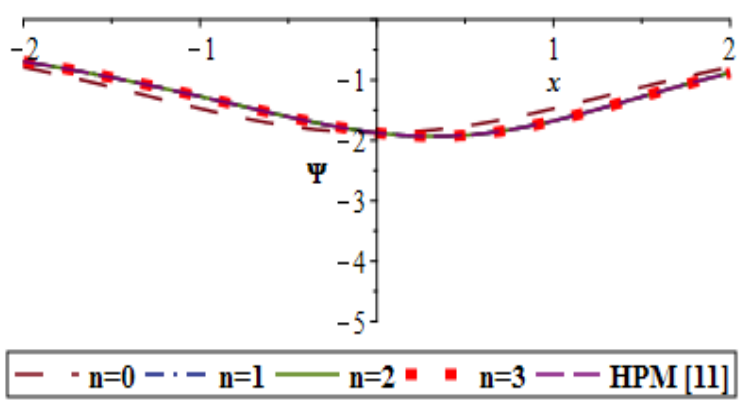

(a)

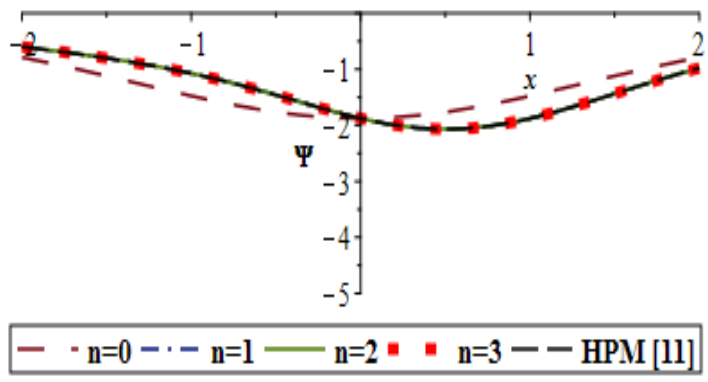

(b)

Figure 8. Comparison plots of $\psi_{F R D T M}$ and $\psi_{H P M}$ [11] of Degasperis-Procesi (DP) equation for $-10 \leq x \leq 10$. (a) $t=0.05, \alpha=1 ;(\mathbf{b}) t=0.1, \alpha=1$.

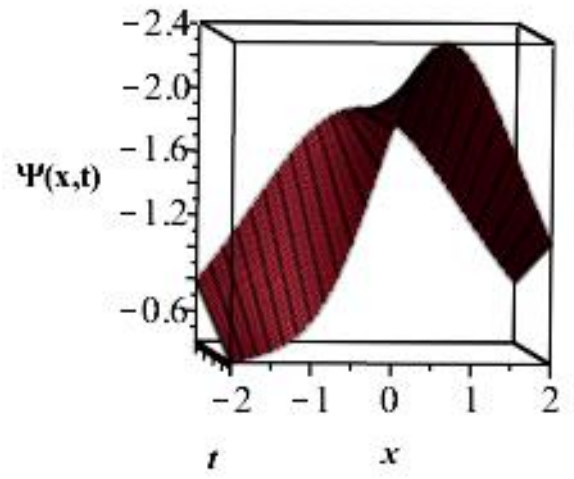

(a)

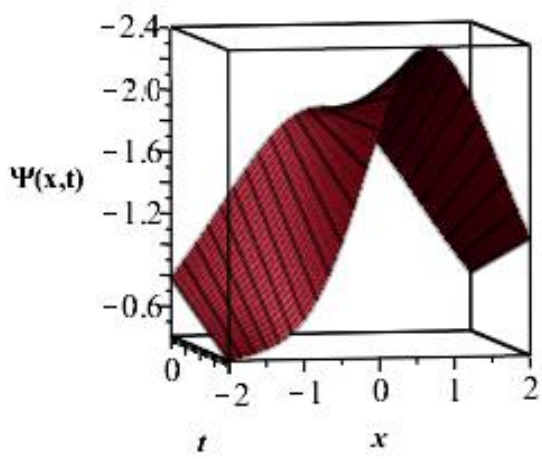

(b)

Figure 9. The solution plots of DP equation at $\alpha=1$. (a) two-terms FRDTM solution (b) HPM solution [11]. 
Table 2. The two term FRDTM approximation results of $\mathrm{CH}$ equation with HPM [28] for $\alpha=1$.

\begin{tabular}{cccccc}
\hline$(x, t)$ & $\psi_{H P M}[28]$ & $\begin{array}{c}\text { FRDTM } \\
(\boldsymbol{n}=\mathbf{0})\end{array}$ & $\begin{array}{c}\text { FRDTM } \\
(\boldsymbol{n}=\mathbf{1})\end{array}$ & $\begin{array}{c}\text { FRDTM } \\
(\boldsymbol{n}=\mathbf{2})\end{array}$ & $\begin{array}{c}\text { FRDTM } \\
(\boldsymbol{n}=\mathbf{3})\end{array}$ \\
\hline$(0.1,0.05)$ & 0.47479025 & 0.45194289 & 0.45194289 & 0.45194289 & 0.45194289 \\
$(0.2,0.05)$ & 0.42913217 & 0.40845903 & 0.40845903 & 0.40845903 & 0.40845903 \\
$(0.4,0.05)$ & 0.35043736 & 0.33351162 & 0.33351162 & 0.33351162 & 0.33351162 \\
$(0.8,0.05)$ & 0.23325678 & 0.22191112 & 0.22191112 & 0.22191112 & 0.22191112 \\
$(-0.2,0.05)$ & 0.42913217 & 0.40845903 & 0.40845903 & 0.40845903 & 0.40845903 \\
$(-0.4,0.05)$ & 0.35043736 & 0.33351162 & 0.33351162 & 0.33351162 & 0.33351162 \\
CPU time & & $0.053 \mathrm{~s}$ & $0.063 \mathrm{~s}$ & $0.078 \mathrm{~s}$ & $0.124 \mathrm{~s}$ \\
\hline
\end{tabular}

Table 3. The two term FRDTM approximation results of $\mathrm{mCH}$ equation with HPM [11] for $\alpha=1$

\begin{tabular}{cccccc}
\hline$(x, t)$ & $\boldsymbol{\psi}_{\text {HPM }}[11]$ & $\begin{array}{c}\text { FRDTM } \\
(\boldsymbol{n}=\mathbf{0})\end{array}$ & $\begin{array}{c}\text { FRDTM } \\
(\boldsymbol{n}=\mathbf{1})\end{array}$ & $\begin{array}{c}\text { FRDTM } \\
(\boldsymbol{n}=\mathbf{2})\end{array}$ & $\begin{array}{c}\text { FRDTM } \\
(\boldsymbol{n}=\mathbf{3})\end{array}$ \\
\hline$(8,0.05)$ & -0.00268298 & -0.00268190 & -0.00268297 & -0.00268298 & -0.00268298 \\
$(9,0.05)$ & -0.00098718 & -0.00098703 & -0.00098718 & -0.00098718 & -0.00098718 \\
$(10,0.05)$ & -0.00036318 & -0.00036316 & -0.00036318 & -0.00036318 & -0.00036318 \\
$(8,0.1)$ & -0.00268406 & -0.00268105 & -0.00268405 & -0.00268406 & -0.00268406 \\
$(9,0.1)$ & -0.00098732 & -0.00098703 & -0.00098732 & -0.00098732 & -0.00098732 \\
$(10,0.1)$ & -0.00036320 & -0.00036316 & -0.00036320 & -0.00036320 & -0.00036320 \\
CPU time & & $0.063 \mathrm{~s}$ & $0.093 \mathrm{~s}$ & $0.078 \mathrm{~s}$ & $0.094 \mathrm{~s}$ \\
\hline
\end{tabular}

Table 4. The two term FRDTM approximation results of DP equation with HPM [11] for $\alpha=1$

\begin{tabular}{cccccc}
\hline$(x, t)$ & $\psi_{\text {HPM }}[11]$ & $\begin{array}{c}\text { FRDTM } \\
(\boldsymbol{n}=\mathbf{0})\end{array}$ & $\begin{array}{c}\text { FRDTM } \\
(\boldsymbol{n}=\mathbf{1})\end{array}$ & $\begin{array}{c}\text { FRDTM } \\
(\boldsymbol{n}=\mathbf{2})\end{array}$ & $\begin{array}{c}\text { FRDTM } \\
(\boldsymbol{n}=\mathbf{3})\end{array}$ \\
\hline$(0,0)$ & -1.875 & -1.8750 & -1.875 & -1.875 & -1.875 \\
$(0.2,0.2)$ & -2.1311490 & -1.8563742 & -2.1311490 & -2.1311490 & -2.1311490 \\
$(0.4,0.4)$ & -2.8273737 & -1.8019555 & -2.8273737 & -2.8273737 & -2.8273737 \\
$(0.8,0.8)$ & -4.7337056 & -4.7337056 & -4.7337056 & -4.7337056 & -4.7337056 \\
$(-0.2,0.2)$ & -1.5815995 & -1.8563742 & -1.5815995 & -1.5815995 & -1.5815995 \\
$(-0.4,0.4)$ & -0.7765374 & -1.8019555 & -0.7765374 & -0.7765374 & -0.7765374 \\
CPU time & & $0.01 \mathrm{~s}$ & $0.016 \mathrm{~s}$ & $0.062 \mathrm{~s}$ & $0.078 \mathrm{~s}$ \\
\hline
\end{tabular}

\section{Conclusions}

In this article, FRDTM is successfully implemented for solving time-fractional $\mathrm{CH}, \mathrm{mCH}$, and DP equations with suitable initial conditions. Solutions are obtained without any transformation and perturbation. Three test problems were performed to validate the precision and efficacy of the current method. Also, it was seen that the obtained results are a good agreement with the solution obtained by Zhang et al. [11,28]. The main benefit of this approach is that linearization is not required for this method and therefore it reduces complex numerical computations significantly compared to the other existing methods such as the perturbation technique, DTM, and ADM. Small size computations over other techniques are the main advantages of the proposed method.

Author Contributions: Each author has contributed equally to prepare and finalize the whole research work of the present paper.

Funding: This research received no external funding.

Acknowledgments: The first author is grateful to the Department of Science and Technology, Govt. of India for providing an INSPIRE fellowship (IF170207) to do the current research.

Conflicts of Interest: The authors declare that there are no conflicts of interests. 


\section{References}

1. Podlubny, I. Fractional Differential Equations; Academic Press: San Diego, CA, USA, 1999.

2. Miller, K.S.; Ross, B. An Introduction to the Fractional Calculus and Fractional Differential Equations; Wiley: New York, NY, USA, 1993.

3. Caputo, M.; Mainardi, F. Linear models of dissipation in an elastic solids. Riv. Nuovo Cim. 1971, 1, 161-198. [CrossRef]

4. Carpinteri, A.; Mainardi, F. Fractals and Fractional Calculus in Continuum Mechanics; Springer: New York, NY, USA, 1997.

5. Jena, R.M.; Chakraverty, S. A new iterative method based solution for fractional Black-Scholes option pricing equations (BSOPE). SN Appl. Sci. 2019, 1, 95. [CrossRef]

6. Baleanu, D.; Diethelm, K.; Scalas, E.; Trujillo, J.J. Fractional Calculus: Models and Numerical Methods; World Scientific Publishing Company: Boston, MA, USA, 2012.

7. Baleanu, D.; Machado, J.A.T.; Luo, A.C. Fractional Dynamics and Control; Springer: Berlin, Germany, 2012.

8. Jena, R.M.; Chakraverty, S. Residual power series method for solving time-fractional model of vibration equation of large membranes. J. Appl. Comput. Mech. 2019, 5, 603-615.

9. He, J.H. Nonlinear oscillation with fractional derivative and its applications. Int. Conf. Vib. Eng. 1998, 98, 288-291.

10. Javeed, S.; Baleanu, D.; Waheed, A.; Khan, M.S.; Affan, H. Analysis of Homotopy Perturbation Method for Solving Fractional Order Differential Equations. Mathematics 2019, 7, 40. [CrossRef]

11. Zhang, B.G.; Li, S.Y.; Liu, Z.R. Homotopy perturbation method for modified Camassa-Holm and Degasperis-Procesi equations. Phys. Lett. A 2008, 372, 1867-1872. [CrossRef]

12. Jena, R.M.; Chakraverty, S. Solving time-fractional Navier-Stokes equations using homotopy perturbation Elzaki transform. SN Appl. Sci 2019, 1, 16. [CrossRef]

13. Singh, J.; Kumar, D.; Kilicman, A. Numerical solutions of nonlinear fractional partial differential equations arising in spatial diffusion of biological populations. Abstr. Appl. Anal. 2014, 535793. [CrossRef]

14. Jena, R.M.; Chakraverty, S.; Jena, S.K. Dynamic Response Analysis of Fractionally Damped Beams Subjected to External Loads using Homotopy Analysis Method. J. Appl. Comput. Mech. 2019, 5, 355-366.

15. Ganji, Z.Z.; Ganji, D.D.; Ganji, A.D.; Rostamian, M. Analytical solution of time-fractional Navier-Stokes equation in polar coordinate by homotopy perturbation method. Numer. Methods Part Differ. Equ. 2010, 26, 117-124. [CrossRef]

16. Yavuz, M.; Ozdemir, N. A Quantitative Approach to Fractional Option Pricing Problems with Decomposition Series. Konuralp J. Math. 2018, 6, 102-109.

17. Edeki, S.O.; Motsepa, T.; Khalique, C.M.; Akinlabi, G.O. The Greek parameters of a continuous arithmetic Asian option pricing model via Laplace Adomian decomposition method. Open Phys. 2018, 16, 780-785. [CrossRef]

18. Wazwaz, A.M. Solitary wave solutions for modified forms of Degasperis-Procesi and Camassa-Holm equations. Phys. Lett. A 2006, 352, 500-504. [CrossRef]

19. Jena, R.M.; Chakraverty, S. Analytical solution of Bagley-Torvik equations using Sumudu transformation method. SN Appl. Sci. 2019, 1, 246. [CrossRef]

20. Kumar, D.; Singh, J.; Kumar, S. Numerical computation of fractional multi-dimensional diffusion equations by using a modified homotopy perturbation method. J. Assoc. Arab Univ. Basic Appl. Sci 2014, 17, $20-26$. [CrossRef]

21. Odibat, Z.; Momani, S. Modified homotopy perturbation method application to quadratic Riccati differential equation of fractional order. Chaos Solitons Fractals 2008, 36, 167-174. [CrossRef]

22. Keskin, Y.; Oturanc, G. Reduced differential transform method: a new approach to fractional partial differential equations. Nonlinear Sci. Lett. A 2010, 1, 61-72.

23. Camassa, R.; Holm, D.D. An integrable shallow water equation with peaked solitons. Phys. Rev. Lett. 1993, 71, 1661-1664. [CrossRef]

24. Gupta, P.K.; Singh, M.; Yildirim, A. Approximate analytical solution of the time fractional Camassa-Holm, modified Camassa-Holm, and Degasperis-Procesi equations by homotopy perturbation method. Sci. Iran. A 2016, 23, 155-165. 
25. Ganji, D.D.; Sadeghi, E.M.M.; Rahmat, M.G. Modified Camassa-Holm and Degasperis-Procesi Equations Solved by Adomian's Decomposition Method and Comparison with HPM and Exact Solutions. Acta Appl. Math. 2008, 104, 303-311. [CrossRef]

26. Lundmark, H.; Szmigielski, J. Multi-peakon solutions of the Degasperis-Procesi equation. Inverse Probl. 2003, 19, 1241-1245. [CrossRef]

27. Degasperis, A.; Procesi, M. Asymptotic Integrability Symmetry and Perturbation Theory; World Scientific: Singapore, 2002; pp. 23-27.

28. Zhang, B.G.; Li, S.Y.; Mao, J.F. Approximate explicit solution of Camassa-Holm equation by He's homotopy perturbation method. J. Appl. Math. Comput. 2009, 31, 239-246. [CrossRef]

(C) 2019 by the authors. Licensee MDPI, Basel, Switzerland. This article is an open access article distributed under the terms and conditions of the Creative Commons Attribution (CC BY) license (http://creativecommons.org/licenses/by/4.0/). 